\title{
Force Concept Inventory: More than just conceptual understanding
}

\author{
Siera M. Stoen $\odot,{ }^{1,2}$ Mark A. McDaniel,,${ }^{1,3,}$ Regina F. Frey $\odot,{ }^{1,4}$ \\ K. Mairin Hynes $\odot{ }^{2}$ and Michael J. Cahill ${ }^{1}$ \\ ${ }^{1}$ Center for Integrative Research on Cognition, Learning and Education, \\ Washington University in St. Louis, One Brookings Drive, St. Louis, Missouri 63130, USA \\ ${ }^{2}$ Department of Physics, Washington University in St. Louis, \\ One Brookings Drive, St. Louis, Missouri 63130, USA \\ ${ }^{3}$ Department of Psychological and Brain Sciences, Washington University in St. Louis, \\ One Brookings Drive, St. Louis, Missouri 63130, USA \\ ${ }^{4}$ Department of Chemistry, Washington University in St. Louis, \\ One Brookings Drive, St. Louis, Missouri 63130, USA
}

(Received 12 July 2019; published 27 January 2020)

\begin{abstract}
The Force Concept Inventory (FCI) can serve as a summative assessment of students' conceptual knowledge at the end of introductory physics, but previous work has suggested that the knowledge measured by this instrument is not a unitary construct. In this article, we consider the idea that FCI performance may reflect a number of student attributes including relational knowledge structures of physics concepts, expertlike attitudes, and problem-solving skills. Using a large calculus-based introductory physics course, we show that knowledge of conceptual relationships (i.e., knowledge structures), attitudinal measures, and problem-solving ability are all measures that uniquely contribute to a postinstruction FCI score. While these associations do not reveal the nature of their relation to the FCI (it could be that good students perform well on all these measures), they do provide evidence that improving any one of these aspects may improve a student's overall FCI score.
\end{abstract}

DOI: 10.1103/PhysRevPhysEducRes.16.010105

\section{INTRODUCTION}

Since its publication in 1992, the Force Concept Inventory (FCI) [1] has been one of the most widely used concept inventories available for introductory physics and is often used as a summative assessment of students' conceptual knowledge at the end of introductory physics. Previous work, however, has suggested that the knowledge measured by this instrument is not a unitary construct [2-8]. Because some instructors and researchers use the FCI as a measure of student learning and teaching effectiveness $[9,10]$ it could be useful to understand the various cognitive and attitudinal aspects, in addition to students' conceptual understanding, that may be associated with performance on the FCI. Consequently, there has been research interest in what the FCI actually measures and what types of knowledge are associated with it $[2-8,11,12]$. In this article, we consider the idea that FCI performance may reflect a number of student attributes including relational knowledge structures of physics

"markmcdaniel@wustl.edu

Published by the American Physical Society under the terms of the Creative Commons Attribution 4.0 International license. Further distribution of this work must maintain attribution to the author(s) and the published article's title, journal citation, and DOI. concepts [13], attitudes [9], and problem-solving skills $[10,13]$. Specifically, the following study explores the extent to which each of these aspects uniquely contributes to a postinstruction FCI score after controlling for general reading and comprehension ability and general math ability. Because we know that knowledge structures [14], attitudes [15], and problem-solving ability [10] develop throughout the semester, this study will assess all of these measures at one time point, postinstruction, to examine how they are related to postinstruction performance on the FCI. In the following subsections we will review the initial literature on how each of these measures relates to the FCI.

\section{A. Associations between learning and attitudinal measures and the FCI}

\section{Knowledge structures and the FCI}

An important goal for many introductory physics courses may be to teach the relationships between concepts in addition to teaching the key concepts. That is, physics students are expected to understand the key concepts from class and how these concepts interrelate, i.e., they have to develop a knowledge structure (knowledge of how the different concepts interrelate) [16]. In addition to helping students perform well on course exams, such structural knowledge might be important for performance on the FCI. 
By definition, because the FCI is a concept inventory, students cannot rely on equations to solve the problems. Instead, students must understand the basic ideas or concepts of physics - they must tap into their knowledge structures-in order to answer the FCI questions. What remains uncertain is the extent to which the relational aspects of the key ideas or concepts (friction, momentum, potential energy, etc.) that are represented in learners' knowledge structures are captured in the FCI.

Theoretically, one might expect that the conceptual relationships represented in physics students' knowledge structures would affect FCI performance. The FCI primarily deals with Newtonian force, a central topic in Newtonian mechanics [11]. The FCI is designed to assess students' underlying understanding and misconceptions about Newtonian force and therefore offers typical student misconceptions as alternative answers in addition to the correct one [11]. As with any problem, the student must first realize what concept(s) are valid for answering the question. Thus, an aspect of students' knowledge that might be associated with the FCI is the organization of, and relationship among, the concepts concerning Newtonian force, that is, the students' knowledge structures. To illustrate how relational knowledge (among concepts) might be involved in answering the FCI questions, consider question 26 shown in Fig. 1. This question tests the relationship between force and velocity. In particular, a common misconception is that force and velocity are proportional or directly related. Another misconception is that force and velocity are not related at all. However, the correct relationship is that force is proportional to change in velocity. Presumably, the nature (index) of how these two topics are related or connected in the student's knowledge structure could reflect these various beliefs or misconceptions.

Malone [13] measured knowledge structures using a card sort task in which students are given sets of cards with problems on them. The students were told not to solve them but group them together based on similarity in solution strategies (i.e., the students were instructed to group them in a meaningful way). Malone [13] reported that the quality of the groupings, as determined by comparison with an expert's groupings, were correlated with the FCI $(r=0.57)$.
However, for research in large-enrollment physics classes, a card-sort task (as used in Malone's [13] study) is not practical. To attempt to model structural knowledge, educators in science, technology, engineering, and mathematics (STEM) courses typically use multiple choice questions, open-ended questions, or concept maps [14]. These methods have drawbacks as well, however. For instance, in multiple choice questions a student can get the correct answer using faulty reasoning. Regarding concept maps, their evaluation can be relatively subjective in nature $[14,17]$. As a first step towards providing a better assessment, researchers have created a more objective way to measure structural knowledge using similarity ratings [18-20]. In this task students rate how similar pairs of key concepts are using a Likert scale. (For more information, see below under study instruments-similarity ratings.) We adopt that methodology in the current study.

Most generally, our interest in measuring structural knowledge and its possible bearing on FCI performance is motivated by a growing body of research in other STEM disciplines that shows the importance of knowledge structures and how they relate to performance [14,19]. More specifically, as far as we know, this is the first study using a similarity rating task, rather than a card-sort task, to measure students' knowledge structures in physics and to examine their relationship to FCI. The inclusion of a student's knowledge structure, constructed through similarity ratings, as a knowledge index is an important addition to the research literature examining students who are more and less expertlike in their physics' knowledge (see Ref. [19] for a similar approach in examining students' introductory statistics knowledge structures, and Ref. [14] for examining students' chemistry knowledge structures).

\section{Expert attitudes and the FCI}

Attitudinal measures, such as the Colorado Learning Attitudes about Science Survey (CLASS) [21], can measure the extent to which one holds expertlike beliefs about, and approaches towards, physics. A growing body of research [15,21-28] shows that more expertlike attitudes are associated with higher learning gains, and these

26. If the woman in the previous question doubles the constant horizontal force that she exerts on the box to push it on the same horizontal floor, the box then moves

1. with a constant speed that is double the speed $v_{0}$ in the previous question.

2. with a constant speed that is greater than the speed $v_{0}$ in the previous question, but not necessarily twice as great.

3. for a while with a speed that is constant and greater than the speed $v_{0}$ in the previous question, then with a speed that increases thereafter.

4. for a while with an increasing speed, then with a constant speed thereafter.

5. with a continuously increasing speed.

FIG. 1. Question 26 from the Force Concept Inventory. 
learning gains are often measured by the FCI or another similar instrument. For example, Ding [29] and Cahill et al. [9] showed that precourse attitudinal measures influenced postconceptual learning, as measured by the FCI. In order to attempt to identify the specific precourse attitudinal measures that related to FCI performance gains, Cahill et al. [9] divided the CLASS into learning approach and solving approach factors (which were similar to factors developed by another group of researchers [30]). The learning approach factor captures the extent to which a student is focused on learning underlying concepts vs specific examples, formulas, or algorithms. The solving approach factor indexes the extent to which students think about and use concepts while they are solving problems. In their work, Cahill et al. [9] found that the learning approach factor was the attitudinal measure most strongly tied to FCI performance gains.

The present study extends the Cahill et al. [9] and others' work [30] and uses the two factors (learning approach and solving approach), instead of an overall CLASS score, to examine whether students' self-reported learning approach and solving approach are associated with a postinstruction FCI score. It is important to note that Cahill et al. [9] used preinstruction CLASS measures to predict learning gains as assessed by the FCI. In contrast, the present study uses postinstruction CLASS measures to gauge the extent to which the FCI reflects a more conceptual approach to learning physics and to solving physics problems (as selfreported). Note that we do not examine gains in FCI from pre- to postinstruction because such gains are indexed relative to each student's pre-instruction performance. Accordingly, gains are not reflective of absolute levels of final performance. Our interest was in examining how absolute levels of performance on the FCI (assessed at the end of the semester) are related to the cognitive and attitudinal aspects targeted herein. We expect both learning approach and solving approach will be related to the FCI. Consider first the learning approach measure of the CLASS. It seems reasonable to expect that if students focus on learning underlying concepts instead of specific examples, then this might relate to how well they perform on conceptually based questions in the FCI (cf. Ref. [31]). Consider next the solving approach measure of the CLASS. Because students cannot rely on equations to solve the problems on the FCI, it is plausible that high performance on the FCI will reflect a solving approach centered on activating relevant concepts.

\section{Problem solving and the FCI}

Physics instruction, especially at the introductory level, focuses heavily on teaching students to solve quantitative physics problems. As theoretical background, we briefly outline a widely received view of problem solving that has clear parallels with quantitative problem solving in physics (the general model was first introduced by Polya [32], based on mathematics problem solving). For problem solving generally, a major process is to understand and represent the critical elements of the problem, and perhaps develop a plan [32,33] (see Ref. [34] for laboratory problems that do not necessarily stimulate planning). In physics problem solving, classic work has shown that effective understanding and representation involves determining what concepts are applicable and how these concepts can be used to plan a solution to the problem $[35,36]$. In the general model, the next process (or stage) is to implement solution steps [37-39]. In physics quantitative problem solving, these processes align with the series of computations that must be carried out [35]. Once an answer is obtained, in the general model another major process is to evaluate whether or not a derived answer is reasonable [32]. This process also aligns with effective problem solving in physics. As one example, instructors may encourage students to evaluate their answers in terms of whether the response is sensible (e.g., in terms of magnitudes of values or particular units). If an answer is not deemed sensible, then the problem can be revisited to check solution steps or to reconstrue the initial understanding of the problem.

The possibility that we explore in the present study is that the concept questions in the FCI involve processes akin to the understanding and representation processes in quantitative problem solving in physics and perhaps the evaluation process of a candidate answer(s). (Cleary, the concept questions are designed to obviate the need to reason through the computational steps; accordingly, we are not insisting that this particular problem-solving process overlaps across quantitative problems and the FCI concept questions.) In other words, though the FCI is not focused on quantitative problem solving per se, we suggest that it might tap into at least some of the general problem-solving skills (e.g., activating relevant constructs to understand the problem and guide and evaluate solutions) that students develop while learning to solve quantitative problems in introductory physics.

For the purposes of this study we will be using a physicsspecific quantitative problem-solving assessment introduced by McDaniel et al. [10] as an independent index of these problem-solving processes. With previously used quantitative problem-solving assessments, there is concern that the problems used in evaluating quantitative problem solving are elementary and do not adequately assess more advanced abilities [10,40]. In contrast, the McDaniel et al. [10] assessment involved multiple quantitative steps and, more importantly, required the students to explicitly break down their problem-solving steps. That is, for each of the three quantitative problems, the students had to (a) generate the key concepts, principles, assumptions, and a plan for solving the problem, (b) execute the plan and reason through the computational work, and (c) evaluate the plausibility of their answer (see Appendix B for the wording of these questions). 
Given that McDaniel et al.'s [10] assessment captures the underlying cognitive skills involved in physics problem solving, it will allow us to examine whether physics problem-solving skills uniquely contribute to FCI performance (postinstruction). Controlling for ACT Math as an index of general quantitative skills [41], we can obtain a look at the underlying general problem-solving skills with computational competence or fluency statistically factored out.

\section{B. Study overview and theoretical hypotheses}

The goal of this study is to explore the degree to which three candidate physics knowledge and attitudinal measures-knowledge of conceptual relationships, attitudinal measures, and physics problem-solving ability—are associated with a postinstruction FCI score. As developed in the preceding sections, we suggest that all these measures play an important role in accounting for student performance on the FCI.

\section{RESEARCH METHODS}

\section{A. Sample}

Out of 711 students enrolled in a Fall 2016 calculusbased introductory physics course at Washington University in Saint Louis, 370 provided informed consent and completed the measures to participate in this study (52\% of the total class population). Of these 370 students, $54 \%$ were female and $46 \%$ were male. Furthermore, $50 \%$ were white, $33 \%$ were Asian, $14 \%$ were underrepresented minorities (defined as African American/Black, Hispanic/ Latinx, Native American/Alaskan Native, or Native Hawaiian/Other Pacific Islander), and 3\% did not report ethnicity data. Additionally, the sample included 34\% engineering majors, $37 \%$ Arts \& Sciences STEM majors (including psychology), and 29\% non-STEM majors. These demographic breakdowns are similar to that of the entire class sample for the Fall 2016 semester. Furthermore, this course is required for all physics, biology, chemistry, earth and planetary science, engineering, material science, and architecture majors, as well as anyone interested in health-oriented training (e.g., medical school).

\section{B. Course curriculum}

The course textbook was Six Ideas That Shaped Physics [42], and the course was designed to develop a deeper understanding of physics concepts and fundamental physics principles as well as develop quantitative problemsolving skills. To achieve this, the course used minilectures and interactive engagement (IE) activities such as demonstrations (including making predictions), two-minute problems, and group work. The two-minute problems were multiple-choice or true-false problems and a majority were taken from the textbook or Mazur's ConcepTests [43]. These two-minute problems were designed to focus on students' misconceptions and conceptual ideas in the course. Depending on the instructor, IE activities represented $40 \%-60 \%$ of class time, and could include some quantitative problem solving. The remainder of class time was spent on minilectures that could include instructor-led examples and derivations. Additionally, the students had online daily homework covering the required readings as well as written weekly homework with multistep quantitative problems. The course spent about $75 \%$ (36 class hours) of the time covering mechanics and the remainder on special relativity. More details about this curriculum can be found in previously published papers $[9,10,15]$.

\section{Study instruments \\ 1. Force concept inventory}

The Force Concept Inventory (FCI) [1] is a 30-question multiple choice test that assesses conceptual knowledge for topics covered in the fall semester (i.e., Newtonian mechanics). Students completed the FCI near the beginning and the end of the semester and the FCI score is the proportion of items a student answered correctly.

\section{Colorado learning attitudes about science survey}

The Colorado Learning Attitudes about Science Survey (CLASS) [21] contains 42 Likert-scale questions anchored at 1 (strongly disagree) and 5 (strongly agree). These items ask about students' agreement with statements related to their perceptions of physics and learning physics, and scores reflect the extent to which students' perceptions match those of experts. Of these 42 items, 5 lack expert consensus and 1 is used to test if the participant is reading the questions, thus only 36 items are used to determine overall CLASS score. In line with previous research [9], 12 of these items (see Appendix A for items) were used to compute a learning approach score and 12 separate items were used to compute a solving approach score. These two scores were used as the primary attitudinal measures in this study. Previous research has shown that students' exam grades and performance on concept inventories are predicted by their degree of expertlike thinking about physics [9]. Students completed the CLASS survey near the beginning and the end of the semester. As mentioned in the introduction, the end-of-semester CLASS survey responses were used for the present study.

\section{Problem-solving assessment}

The problem-solving assessment [10] includes three problems, created by one of the course instructors in Fall 2009, which all relate to conservation of energy. This assessment has been used periodically in the introductory physics class since 2009. Starting in Fall 2015, the assessment was modified slightly: Students were explicitly instructed to provide a diagram (previously they had not been required to do so). Because the course has changed 
slightly throughout the years, we believed that for Fall 2016 one of the problems (problem two-see Appendix B) was not taught sufficiently and accordingly that problem was excluded from the present analyses. Each problem contains three subquestions designed to demonstrate students' ability to (a) generate the key concepts, principles, assumptions, and a plan for solving the problem, including generating a diagram, (b) execute the plan and reason through the computational work, and (c) evaluate the plausibility of their answer (see Appendix B for the wording of these questions).

For scoring purposes, a highly reliable rubric was constructed to directly evaluate the problem-solving processes elicited (i.e., subquestions a, b, and c for each problem). Additionally, a total score combining all problem-solving processes for problems one and three was computed to provide an overall summary of problemsolving performance. The total scores range from 0 to 9 , and more details about this problem-solving assessment, rubric, and total score can be found in previously published research [10]. Students completed the problem-solving assessment near the beginning and the end of the semester. The present study focused on the end-of-semester problemsolving assessment.

\section{Similarity ratings}

Students completed a similarity rating task, which is used to construct students' knowledge structures and then these structures are compared to those of experts. For this task, a set of 15 key physics concepts was developed with input from three physics experts who taught or worked with the introductory physics series. First, two different introductory physics instructors' independently generated lists of key physics terms. Second, a third expert combined the lists into one that consisted of only overlapping key terms. This list was then checked to make sure all topics were covered in the course, resulting in the final list of $15 \mathrm{key}$ terms (see Appendix C for a list of concepts). Using 15 terms is consistent with previous research $[14,19,44]$.

Toward the end of the first semester of introductory physics, students rated the similarity between all possible pairs of these 15 concepts, resulting in 105 total relatedness judgments on a scale from 1 (highly unrelated) to 7 (highly related). To ensure that students utilized the full scale, they were presented with the list of key terms before completing the task and were asked to choose both a highly related and highly unrelated pair of terms to serve as anchors (see Appendix $\mathrm{C}$ for similarity task instructions). After completing the entire rating task, each student's matrix of similarity ratings was entered into Pathfinder [45], which is an open-source software developed by Schvaneveldt [44-46] that takes a matrix of similarity ratings as input and produces a network representing the relatedness among these key terms. This network is a spatial representation of relatedness among the items, with less related items separated by larger distances.

To produce a network, Pathfinder uses two parameters $q$ and $r$ to search the terms (nodes) and find the closest direct path. A link only remains if it is the most direct path between two concepts. The length of a path is defined by the $r$ parameter which has an inverse relationship with the number of displayed links. As $r$ decreases, links are added to the network; when $r=\infty$, the number of links in a network is minimized. The maximum number of links allowed and the density of the network is defined by the parameter $q$. Keeping with previous literature, we chose parameter values which generate the simplest network in Pathfinder [45], which occurs when $r=\infty$ and $q=n-1$, where $n$ is equal to the number of terms [14].

Typically, these networks are then compared to an "expert network," which is an average of multiple experts' networks, in order to determine how similar one's network is to the average expert network. One index of network similarity is path length correlation (PLC), which is a measure of how the between-concept distances in one network correlate with those same distances in another network (usually an expert network). A second index, neighborhood similarity (NS), measures the similarity in how nodes are grouped. In essence, NS captures the similarity between networks in terms of which concepts or nodes are clustered together. Both PLC and NS range from 0 to 1 . In addition to these two quantitative measures, Pathfinder also generates a visual representation of a student's network of these concepts-revealing what connections students are making, what misconceptions they may have about the material, and the connections or similarities between key concepts that they are missing (See Sec. IV for example representations).

For this study, three professors at Washington University in Saint Louis, who have taught or are currently teaching introductory physics, made similarity ratings on the 15 key terms, and Pathfinder networks were produced. The internal consistency, or coherence, of each individual network was then checked, with all achieving acceptable levels of coherence ( $\geq 0.2$ [14]; see Table I). The experts' networks were then averaged by the Pathfinder program to produce an average expert network. As a check that the average expert network was representative of individual experts, each individual expert network was then compared to this average network to make sure each had acceptable PLC

TABLE I. The coherence and consistency of each expert's network with the average-expert network (averaged across the three experts).

\begin{tabular}{lccc}
\hline \hline & Expert 1 & Expert 2 & Expert 3 \\
\hline Coherence & 0.325 & 0.384 & 0.426 \\
PLC & 0.804 & 0.883 & 0.890 \\
NS & 0.771 & 0.659 & 0.758 \\
\hline \hline
\end{tabular}




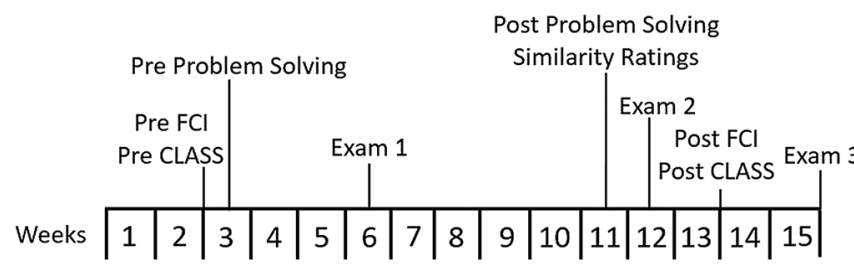

FIG. 2. Visual timeline of the study procedures.

$(>0.5$ [47]) and NS (>0.4 [14,44]) levels with respect to the average network. As shown in Table I, all experts had acceptable PLC and NS levels, so the average network from the three experts was retained and used as the comparison expert network in computing PLC and NS scores for individual students.

\section{Procedure}

Students who completed both the FCI and CLASS were allowed to replace one nonzero lab grade with a perfect score and slightly less than $1 \%$ course credit was given for completing the similarity rating task and problem-solving assessment. All instruments were completed online, except the problem-solving assessment, which was completed on paper during lab. The FCI and CLASS were completed during the first 2-3 weeks of the 15-week semester and again during weeks 13 and 14. The problem-solving assessment was completed during week 3 and again during week 11. Pathfinder was completed during week 11; this was 3 weeks after all key topics on the task were covered in the course. Figure 2 shows a visual timeline.

\section{E. Analytic strategy}

The main goal of this study was to explore what specific knowledge and attitudinal measures are associated with postinstruction FCI performance. First, we computed zero-order correlations between all of the variables to determine the extent of overlap. Next, we conducted a hierarchical multiple regression with postinstruction FCI as our dependent measure. In order to control for general comprehension or reading ability and quantitative skills, step 1 of the hierarchical multiple regression included both ACT Math and ACT Verbal. These are standardly used to control for general reading and quantitative skills, respectively [41]. Step 2 added PLC and NS (measures of poststructural knowledge) as predictors; step 3 added postattitudinal measures (solving approach and learning approach) as predictors; finally, step 4 added post-problem-solving ability as a predictor. At each step, the regression coefficients indicate how strong the relationship is between each predictor and the dependent measure, once the influence of all other predictors have been taken into account. In particular, for every $1 \mathrm{SD}$ change in the predictor, the dependent measure changes by the proportion of a SD indicated by the coefficient. Additionally, after each step we provide the $R^{2}$ and test whether the change is statistically significant.

\section{RESULTS}

\section{A. Correlations}

Table II shows the Pearson correlations $(r)$ between all the independent (i.e., knowledge of conceptual relations, attitudes, and physics problem-solving ability), and dependent (i.e., FCI) measures. Correlations measure the association between two continuous variables. The traditional convention is that 0.1 indicates a small correlation, 0.3 indicates a medium correlation, and 0.5 indicates a large correlation [47]. All correlations with the FCI reached significance $(p<0.05)$ and all primary measures (i.e., PLC, NS, CLASS learning approach, CLASS solving approach, and problem solving) had medium correlations with the FCI. Additionally, both our ACT variables (i.e., ACT Math and ACT Verbal) had a small correlation with the FCI. Overall, this pattern indicates that all of our predictor variables were related to FCI performance, but each was not redundant with conceptual knowledge as measured by the FCI.

TABLE II. Pearson correlations between all the independent and dependent measures. Superscripts indicate $p$ values. FCI, PLC, NS, attitudinal learning approach, attitudinal solving approach, and problem solving are postinstruction measures. ACT Math and ACT Verbal were used to control for general comprehension or reading ability and quantitative skills, respectively.

\begin{tabular}{lccccccc}
\hline \hline & ACT Verbal & ACT Math & Learning Approach & Solving Approach & Problem Solving & PLC & NS \\
\hline FCI & $0.180^{\mathrm{c}}$ & $0.254^{\mathrm{c}}$ & $0.432^{\mathrm{c}}$ & $0.315^{\mathrm{c}}$ & $0.356^{\mathrm{c}}$ & $0.462^{\mathrm{c}}$ & $0.305^{\mathrm{c}}$ \\
ACT Verbal & $\ldots$ & $0.235^{\mathrm{c}}$ & 0.102 & -0.004 & $0.146^{\mathrm{b}}$ & $0.172^{\mathrm{c}}$ & $0.143^{\mathrm{b}}$ \\
ACT Math & & $\ldots$ & $0.244^{\mathrm{c}}$ & $0.105^{\mathrm{a}}$ & $0.200^{\mathrm{c}}$ & $0.241^{\mathrm{c}}$ & $0.157^{\mathrm{b}}$ \\
Learning approach & & & $\ldots$ & $0.449^{\mathrm{c}}$ & $0.258^{\mathrm{c}}$ & $0.262^{\mathrm{c}}$ & $0.146^{\mathrm{b}}$ \\
Solving approach & & & & $\ldots$ & $0.115^{\mathrm{a}}$ & $0.207^{\mathrm{c}}$ & $0.176^{\mathrm{c}}$ \\
Problem solving & & & & & $\ldots .263^{\mathrm{c}}$ & $0.193^{\mathrm{c}}$ \\
PLC & & & & & & $\ldots .621^{\mathrm{c}}$ \\
\hline \hline
\end{tabular}

\footnotetext{
${ }_{b}^{\mathrm{a}} p<0.05$

${ }^{\mathrm{b}} p<0.01$

${ }^{\mathrm{c}} p<0.001$
} 
TABLE III. Regression-model summaries predicting postinstruction conceptual knowledge as measured by the FCI from similarity ratings (PLC and NS), attitudinal measures (learning approach and solving approach), and problem solving ability after controlling for general reading and quantitative skills (i.e., ACT verbal and ACT Math). Superscripts indicate $p$ values. Measures of general reading and mathematical ability were added in step 1, PLC and NS in step 2, attitudinal measures in step 3, and problem solving ability in step 4 . The model level statistics show the $R^{2}$ for each model and the change in $R^{2}$ between each step $\left(\Delta R^{2}\right)$.

\begin{tabular}{|c|c|c|c|c|}
\hline & \multicolumn{4}{|c|}{ Standardized predictor coefficients } \\
\hline & Step 1 & Step 2 & Step 3 & Step 4 \\
\hline ACT Verbal & $0.127^{\mathrm{a}}$ & 0.075 & 0.076 & 0.063 \\
\hline ACT Math & $0.224^{\mathrm{c}}$ & $0.135^{\mathrm{b}}$ & 0.077 & 0.060 \\
\hline PLC & & $0.401^{\mathrm{c}}$ & $0.322^{\mathrm{c}}$ & $0.295^{\mathrm{c}}$ \\
\hline NS & & 0.024 & 0.023 & 0.014 \\
\hline Learning approach & & & $0.265^{\mathrm{c}}$ & $0.230^{\mathrm{c}}$ \\
\hline Solving approach & & & $0.117^{\mathrm{a}}$ & $0.121^{\mathrm{a}}$ \\
\hline \multirow[t]{2}{*}{ Problem solving } & & & & $0.181^{\mathrm{c}}$ \\
\hline & \multicolumn{4}{|c|}{ Model level statistics } \\
\hline$R^{2}$ & $0.080^{\mathrm{c}}$ & $0.241^{\mathrm{c}}$ & $0.341^{\mathrm{c}}$ & $0.369^{c}$ \\
\hline$\Delta R^{2}$ & & $0.161^{\mathrm{c}}$ & $0.100^{\mathrm{c}}$ & $0.028^{\mathrm{c}}$ \\
\hline $\begin{array}{l}{ }_{\mathrm{a}}^{\mathrm{a}} p<0.05 \\
{ }_{p}<<0.01 \\
{ }^{\mathrm{c}} p<0.001\end{array}$ & & & & \\
\hline
\end{tabular}

\section{B. Regression analyses}

Table III shows the results of a hierarchical regression model that was conducted to determine what measures uniquely predicted postinstruction FCI performance. Postinstruction conceptual knowledge, as measured by the FCI, was used as the dependent measure.

Step 1 shows that both ACT Math and ACT Verbal were predictors of the FCI. However, as additional predictors reflecting various aspects of physics knowledge (i.e., knowledge of conceptual relations, expertlike attitudes, problem-solving ability) were added into the model, ACT Math and ACT Verbal dropped out as predictors. Step 2 shows that the addition of PLC and NS further improved the model, though NS was not a significant predictor. Consistent with previous literature [14], PLC has a stronger association with performance measures. Step 3 shows that attitudinal learning approach was a stronger predictor than attitudinal solving approach. Further, in step 4 the addition of problem-solving ability significantly improved the model. Examination of the standardized regression coefficients in step 4 shows that PLC was the strongest predictor of the FCI, followed by attitudinal learning approach and problem-solving ability. These results indicate that several different aspects of development of physics' knowledge (knowledge of conceptual relations, attitudes, and problem-solving ability) contributed to the FCI. Further, these patterns show that knowledge of conceptual relations, particularly PLC, accounted for a significant amount of variance in the FCI. Indeed, even after controlling for all of the other predictors, PLC remained as the most robust predictor of FCI performance.

\section{DISCUSSION}

This study showed that a student's knowledge of conceptual relationships (i.e., knowledge structure), attitudinal measures, and physics problem-solving ability are all independently associated with postinstruction FCI performance. This finding is in agreement with our hypothesis that the FCI reflects a combination of several, perhaps interrelated, measures: knowledge of conceptual relations, self-reported expertlike attitudes, and physics problemsolving ability. Furthermore, the assessment of a student's knowledge structure, constructed through similarity ratings (by Pathfinder analysis), as a knowledge index is an important addition to the research literature examining students who are more and less expertlike in their physics' knowledge. We amplify on this point below.

\section{A. Different measures associated with the FCI \\ 1. Knowledge structures}

One of our more novel findings is that a student's relational knowledge structure is associated with FCI performance and is the strongest predictor of FCI performance relative to the other measures we examined (as captured by the standardized beta weights). This can be thought of as the mental structure of how students connect topics together (i.e., a mental concept map [14]). This knowledge structure reflects a student's perception of how related (or unrelated) topics are to each other and can be formally compared to that of an expert.

Furthermore, as we previously mentioned, in addition to the two quantitative measures (PLC and NS) generated by the Pathfinder Program, Pathfinder also generates a visual representation of a student's network of these concepts. One revealing feature of the Pathfinder visual representation, which the FCI does not give, is an explicit rendering of how the students relate important topics. Visual inspection of the generated structure would allow an instructor to critically think about how the material is being interpreted by students and what misconceptions a student may have. Instructors could then further consider how misinterpretations might be potentially avoided or corrected through modifications to how the topics are taught.

For example, below in Figs. 3(a) and 4(a) are visual renderings of two students' Pathfinder networks. Concepts that are more highly related have shorter links than concepts that are unrelated. Comparing linkages of key physics concepts in Fig. 3(a), it can be seen that they highly overlap with the experts' knowledge structure [shown in both Figs. 3(b) and 4(b)]. This student received both a high 

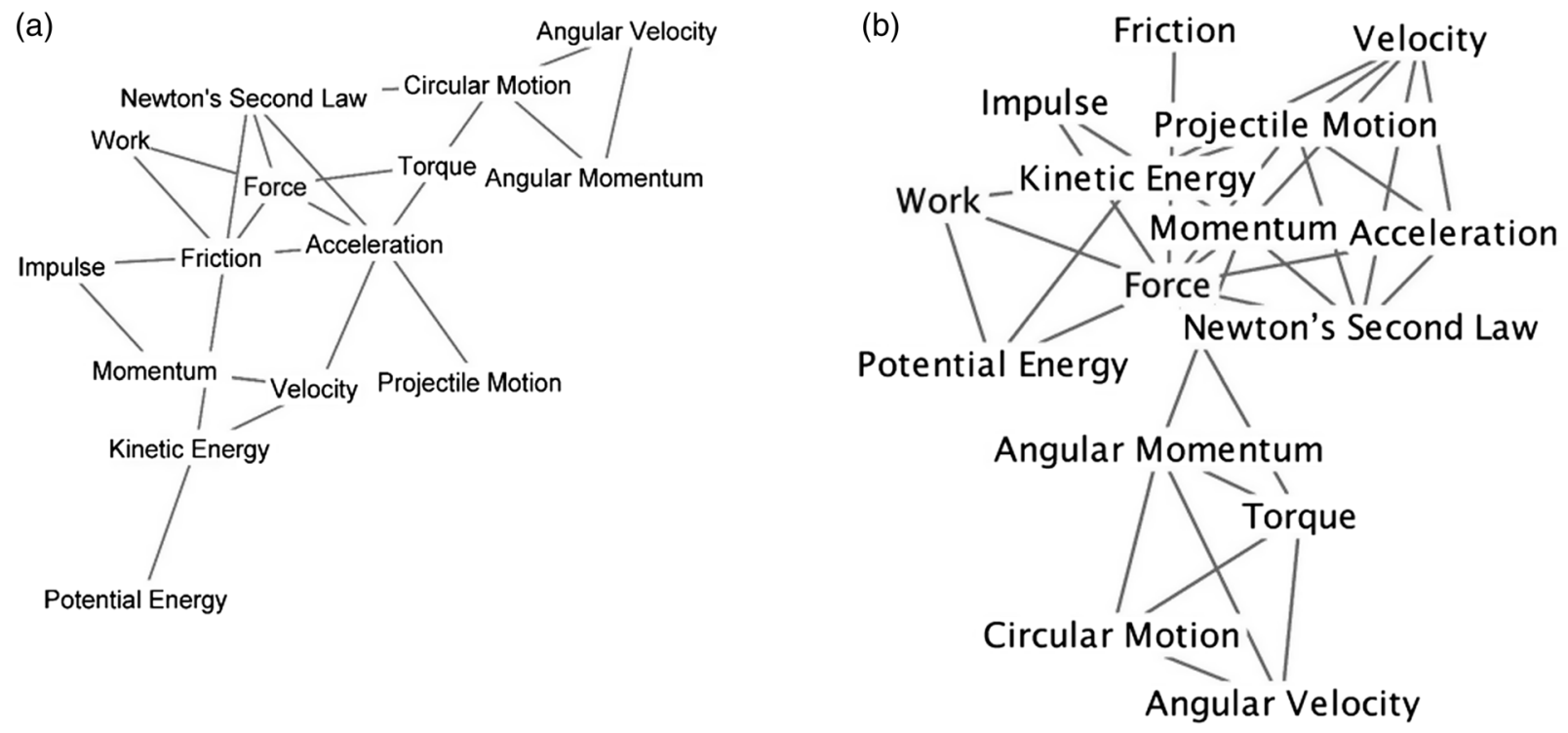

FIG. 3. Sample student Pathfinder network that had both a high FCI score and a high PLC score (a) and experts' Pathfinder network (b).

FCI score (0.87) and a high PLC score (0.73); nevertheless, and importantly for the present point, from their network one can see that they still misunderstand the relationship between potential energy and work and the connection between potential energy and kinetic energy. Both of these connections are important for solving conservation of mechanical energy problems.

Next consider Fig. 4(a), which differs significantly from the average expert network. This student received both a low FCI (0.13) and low PLC score (0.41). While there are numerous misconceptions evident, the most problematic may be those related to energy. There is no connection between kinetic energy and potential energy and neither of

(a)

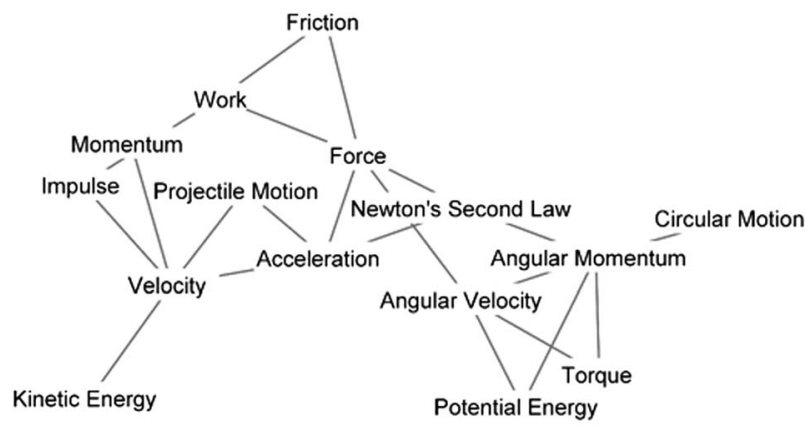

those are connected to work. Furthermore, work is very far away from kinetic energy and potential energy, which says that the student thinks them highly unrelated. Overall this student learned the most obvious connections (e.g., force and acceleration, acceleration and velocity) but not the deeper ones (e.g., momentum and force).

\section{Expert attitudes}

Consistent with previous literature [9], one attitudinal measure that is associated with the FCI is students' selfreported learning approach, as assessed by the CLASS. A high learning approach score indicates that a student focuses on or learns the underlying theories or concepts (b)

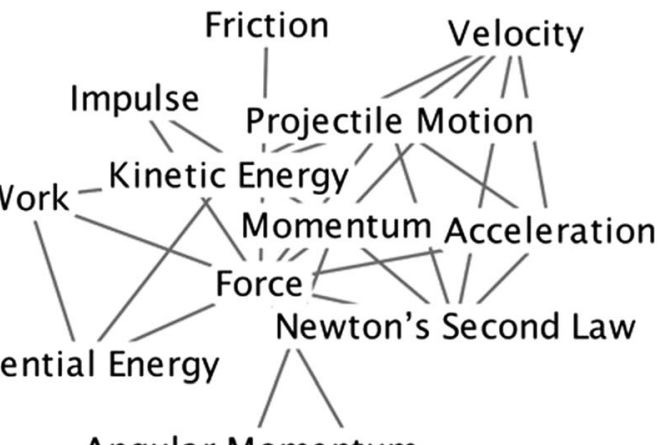

Angular Momentum

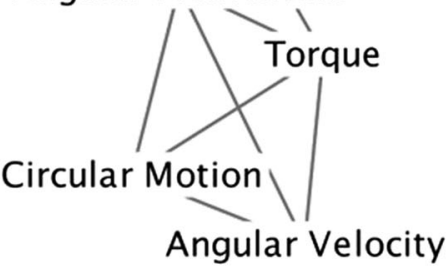

FIG. 4. Sample student Pathfinder network that had both a low FCI score and a low PLC score (a) and experts' Pathfinder network (b). 
rather than a rote memorization approach in acquiring physics knowledge. A novel finding is that the attitudinal measure "solving approach" was also associated with the FCI. A high solving-approach score indicates that a student takes a more concept-based approach to solving problems instead of an algorithmic approach. These students approach a problem using the underlying theories instead of simply memorizing examples. This is, arguably, the kind of knowledge that is needed to do well on the FCI, which is a conceptual inventory.

It is perhaps noteworthy that a previous study [9], which used pre-instruction CLASS measures to predict learning gains as assessed by the FCI, did not find that solving approach uniquely predicted FCI. Unlike that previous study, the current study used postinstruction CLASS measures to gauge the extent to which FCI reflects expertlike beliefs and approaches towards physics. Furthermore, we did not use gains because those are relative to the student's initial level of performance, and thus do not gauge absolute levels of knowledge achieved by any particular student. Our interest in this study was examining what indices are related to absolute levels of performance on the FCI. That being said, one pattern that was consistent in both studies is that expertlike attitudes were an important measure in relating to FCI performance.

\section{Problem solving}

Another measure that is associated with the FCI is physics problem-solving ability. The more successful students in physics tend to problem solve using a principled approach (i.e., they first consider what major principle(s) or concepts(s) can be applied to solve the problem(s) [48-53]). This is in contrast to students who use a more formulaic approach (i.e., they look at equations that match the quantities and unknowns and algebraically manipulate them in order to solve the problem(s) [48-53]). Previous literature [53] has shown that novices take a more formulaic approach towards problem solving. Although taking a formulaic approach in problem solving is fairly effective at obtaining the correct numerical solution it fails to bring in the role that principles or concepts have in solving problems. Furthermore, it fails to promote conceptual understanding and long-term retention of important concepts in physics [53]. In agreement with prior work [48-53] in the introduction we adopted a general problem-solving model that assumes there are conceptual knowledge aspects of successful quantitative problem solving. Because the quantitative problem-solving assessment we administered (see Appendix B) first required the students to come up with key physics principles they were going to use and justify their choices, we encouraged students to determine what concepts were relevant and valid within a particular problem and why. Our results support the idea that these important problem-solving processes are involved in FCI performance, and accordingly, the FCI might help index the development of this aspect of physics problem solving. In a sense, the conceptual questions on the FCI might be thought of as problems.

\section{B. Study limitations}

One limitation of this study is that the data came from a single, selective research university. Hence, the results may not generalize to institutions with different student characteristics, populations, and course content. Future research should investigate whether the results replicate at other universities. Further, because the CLASS and FCI were not a required component of the course, participation was likely not random, with certain types of students (e.g., highly conscientious students) possibly overrepresented in the sample. Because of the fact that our samples may not have been completely representative of the populations from which they were drawn, we acknowledge the possibility that our results may not be representative of all of the students in these courses $(52 \%$ of the overall course population is represented in our sample). In order to see if our sample showed signs of being not representative, we calculated the standard deviation of the post-instruction FCI score and found it to be 0.19 . This is consistent with previously published literature of 0.21 [15]. Furthermore, the average FCI score of our sample, which included everyone who took the FCI, was 0.59 and the average final course grade for our sample was a B, which was identical to that of the whole class. Additionally, we have no a priori reason to suspect that the variables that influence one's likelihood of participating in the study would influence the associations between different learning and attitudinal measures.

\section{Conclusion}

This study showed that knowledge of conceptual relationships (i.e., knowledge structures), attitudinal measures, and problem-solving ability are all independently associated with postinstruction FCI performance. While these associations do not reveal the nature of their relation to the FCI (it could be that good students perform well on all these measures), they do provide evidence that improving any one of these aspects may improve a student's overall FCI score. Further research should look at whether or not increased focus on one aspect (i.e., knowledge of conceptual relations, expertlike attitudes, or problem-solving ability) leads to an increase in conceptual knowledge as measured by the FCI. Moreover, the inclusion of a student's knowledge structure, generated by Pathfinder, as a knowledge index, is an important addition to the research literature examining students who are more and less expertlike in their physics knowledge.

\section{ACKNOWLEDGMENTS}

We would like to thank the students and professors of the Fall introductory physics course for participating in this 
study. Furthermore, a special thanks to Merita Haxhia and Mairin Hynes for administering the assessments and Angela Fink for her linguistic expertise.

\section{APPENDIX A: TWO-FACTOR STRUCTURE (LISTED BY CLASS ITEM NUMBER)}

Learning approach:

1. A significant problem in learning physics is being able to memorize all the information I need to know.

5. After I study a topic in physics and feel that I understand it, I have difficulty solving problems on the same topic.

6. Knowledge in physics consists of many disconnected topics.

8. When I solve a physics problem, I locate an equation that uses the variables given in the problem and plug in the values.

10. There is usually only one correct approach to solving a physics problem.

12. I cannot learn physics if the teacher does not explain things well in class.

17. Understanding physics basically means being able to recall something you've read or been shown.

20. I do not spend more than five minutes stuck on a physics problem before giving up or seeking help from someone else.

21. If I don't remember a particular equation needed to solve a problem on an exam, there's nothing much I can do (legally!) to come up with it.

22. If I want to apply a method used for solving one physics problem to another problem, the problems must involve very similar situations.

34. I can usually figure out a way to solve physics problems.

40. If I get stuck on a physics problem, there is no chance I'll figure it out on my own.

Solving approach:

2. When I am solving a physics problem, I try to decide what would be a reasonable value for the answer.

11. I am not satisfied until I understand why something works the way it does.

15. If I get stuck on a physics problem on my first try, I usually try to figure out a different way that works.

23. In doing a physics problem, if my calculation gives a result very different from what I'd expect, I'd trust the calculation rather than going back through the problem.

24. In physics, it is important for me to make sense out of formulas before I can use them correctly.

26. In physics, mathematical formulas express meaningful relationships among measurable quantities.

29. To learn physics, I only need to memorize solutions to sample problems.
32. Spending a lot of time understanding where formulas come from is a waste of time.

36. There are times I solve a physics problem more than one way to help my understanding.

37. To understand physics, I sometimes think about my personal experiences and relate them to the topic being analyzed.

39. When I solve a physics problem, I explicitly think about which physics ideas apply to the problem.

42. When studying physics, I relate the important information to what I already know rather than just memorizing it the way it is presented.

\section{APPENDIX B: PROBLEM-SOLVING ASSESSMENT}

Problem 1:

As part of the San Francisco police department's forensic team, you are investigating a potential crime scene where a pedestrian was struck by a concrete cylinder that originated from a construction site at the top of a $45 \mathrm{~m}$ high hill. The investigation reveals that the cylinder was moving at $40 \mathrm{~m} / \mathrm{s}$ when it struck a pedestrian. Some quick measurements reveal that the thin-walled cylinder is $3.2 \mathrm{~m}$ long, has an outer radius of $2.0 \mathrm{~m}$, and a wall thickness of $5.0 \mathrm{~cm}$. Just to cover all the bases, you look up the density of concrete and note it is about $2800 \mathrm{~kg} / \mathrm{m}^{3}$. In your report, will you conclude that this was an accident that resulted when the cylinder was jostled loose or will you conclude that this was a premeditated attempt on the person's life?

(a) Briefly explain with words and/or drawings how you would use physics to answer this question. Be sure to include key physics principles you plan to use and any assumptions/approximations that you will make.

(b) Now execute your plan of attack and determine conclusively whether or not this was an accident. Show your work and explain your reasoning.

(c) Considering the magnitude, sign, and units of your result in part (b), discuss the plausibility of your result.

Problem 2:

In a volcanic eruption, a $2.0 \mathrm{~kg}$ piece of porous rock is thrown straight upward with an initial speed of $40 \mathrm{~m} / \mathrm{s}$. It travels upward a distance of $50 \mathrm{~m}$ before it begins to fall back to earth. If the increase in thermal energy due to air resistance on the way down is $70 \%$ of that on the way up, determine the speed of the rock when it returns to its original position.

(a) Identical to Problem 1

(b) Now execute your plan of attack. Show your work and explain your reasoning

(c) Identical to Problem 1.

Problem 3:

A block is released from rest at a height $d$ and slides down a frictionless ramp and onto a first plateau, which has 
a length $d$ and where the coefficient of kinetic friction is 0.50 . If the block is still moving, it then slides down a second frictionless ramp through a height of $d / 2$ and onto a lower plateau, which has a length of $d / 2$ and where the coefficient of kinetic friction is again 0.50. If the block is still moving, it then slides up a frictionless ramp until it momentarily stops. Use energy concepts to determine where the block ultimately comes to rest. If it stops on a plateau, specify which one and the distance from the left edge of the plateau. If it stops (momentarily) on the ramp, specify its height above the lower plateau when it comes to rest.

(a), (b), and (c) are identical to those in problem 2.

\section{APPENDIX C: SIMILARITY TASK INSTRUCTIONS \& KEY TERMS}

The following survey asks you to judge on a scale of 1-7 how related the following 15 terms are to each other:

1. Momentum
2. Angular momentum

3. Work

4. Kinetic energy

5. Potential energy

6. Force

7. Torque

8. Friction

9. Impulse

10. Velocity

11. Acceleration

12. Angular velocity

13. Newton's second law

14. Circular motion

15. Projectile motion

Before continuing, please pick both a highly related and an unrelated pair to serve as anchors. Remember to use the full range of the scale when making your ratings. A 1 indicates that the pair is highly unrelated and a 7 indicates that the pair is highly related.
[1] D. Hestenes, M. Wells, and G. Swackhamer, Force Concept Inventory, Phys. Teach. 30, 141 (1992).

[2] D. Huffman and P. Heller, What does the Force Concept Inventory actually measure?, Phys. Teach. 33, 138 (1995).

[3] N. Lasry, S. Rosenfield, H. Dedic, A. Dahan, and O. Reshef, The puzzling reliability of the Force Concept Inventory, Am. J. Phys. 79, 909 (2011).

[4] J. Stewart, C. Zabriskie, S. DeVore, and G. Stewart, Multidimensional item response theory and the Force Concept Inventory, Phys. Rev. Phys. Educ. Res. 14, 010137 (2018).

[5] T. F. Scott, D. Schumayer, and A. R. Gray, Exploratory factor analysis of a Force Concept Inventory data set, Phys. Rev. ST Phys. Educ. Res. 8, 020105 (2012).

[6] M. R. Semak, R. D. Dietz, R. H. Pearson, and C. W. Willis, Examining evolving performance on the Force Concept Inventory using factor analysis, Phys. Rev. Phys. Educ. Res. 13, 010103 (2017).

[7] J. Wang and L. Bao, Analyzing Force Concept Inventory with item response theory, Am. J. Phys. 78, 1064 (2010).

[8] R. P. Springuel, M. C. Wittmann, and J. R. Thompson, Applying clustering to statistical analysis of student reasoning about two-dimensional kinematics, Phys. Rev. ST Phys. Educ. Res. 3, 020107 (2007).

[9] M. J. Cahill, M. A. McDaniel, R. F. Frey, K. M. Hynes, M. Repice, J. Zhao, and R. Trousil, Understanding the relationship between student attitudes and student learning, Phys. Rev. ST Phys. Educ. Res. 14, 010107 (2018).

[10] M. A. McDaniel, S. M. Stoen, R. F. Frey, Z. E. Markow, K. M. Hynes, J. Zhao, and M. J. Cahill, Dissociative conceptual and quantitative problem-solving outcomes across interactive engagement and traditional format introductory physics, Phys. Rev. Phys. Educ. Res. 12, 020141 (2016).

[11] D. Hestenes and I. Halloun, Interpreting the Force Concept Inventory: A response to March 1995 critique by Huffman and Heller, Phys. Teach. 33, 502 (1995).

[12] A. Savinainen and P. Scott, The Force Concept Inventory: A tool for monitoring student learning, Phys. Educ. 37, 45 (2002).

[13] K. L. Malone, Correlations among knowledge structures, Force Concept Inventory, and problem-solving behaviors, Phys. Rev. ST Phys. Educ. Res. 4, 020107 (2008).

[14] K. Y. Neiles, I Todd, and D. M. Bunce, Establishing the validity of using network analysis software for measuring students' mental storage of chemistry concepts, J. Chem. Educ. 93, 821 (2016).

[15] M. J. Cahill, K. M. Hynes, R. Trousil, L. A. Brooks, M. A. McDaniel, M. Repice, J. Zhao, and R. F. Frey, Multiyear, multi-instructor evaluation of a large-scale interactiveengagement curriculum, Phys. Rev. ST Phys. Educ. Res. 10, 020101 (2014).

[16] D. Sands, M. Parker, H. Hedgeland, S. Jordan, and R. Galloway, Using concept inventories to measure understanding, High. Educ. Pedagog. 3, 173 (2018).

[17] J. R. McClure, B. Sonak, and H. K. Suen, Concept map assessment of classroom learning: Reliability, validity, and logistical practicality, J. Res. Sci. Teach. 36, 475 (1999).

[18] W. H. Acton, P. J. Johnson, and T. E. Goldsmith, Structural knowledge assessment: Comparison of referent structures, J. Educ. Psychol. 86, 303 (1994).

[19] T. E. Goldsmith, P. J. Johnson, and W. H. Acton, Assessing structural knowledge, J. Educ. Psychol. 83, 88 (1991). 
[20] P. J. Johnson, T. E. Goldsmith, and K. W. Teague, Locus of the predictive advantage in pathfinder-based representations of classroom knowledge, J. Educ. Psychol. 86, 617 (1994).

[21] K. K. Perkins, W. K. Adams, S. J. Pollock, N. D. Finkelstein, and C.E. Wieman, Correlating student beliefs with student learning using the Colorado Learning Attitudes about Science Survey, AIP Conf. Proc. 790, 61 (2005).

[22] E. F. Redish, J. M. Saul, and R. N. Steinberg, Student expectations in introductory physics, Am. J. Phys. 66, 212 (1998).

[23] V. K. Otero and K.E. Gray, Attitudinal gains across multiples universities using the Physics and Everyday Thinking curriculum, Phys. Rev. ST Phys. Educ. Res. 4, 020104 (2008).

[24] E. Brewe, L. Kramer, and G. O'Brien, Modeling instruction: Positive attitudinal shifts in introductory physics measured with CLASS, Phys. Rev. ST Phys. Educ. Res. 5, 013102 (2009).

[25] E. Brewe, A. Traxler, J. de la Garza, and L. Kramer, Extending positive CLASS results across multiple instructors and multiple of Modeling Instruction, Phys. Rev. ST Phys. Educ. Res. 9, 020116 (2013).

[26] B. A. Lindsey, L. Hsu, H. Sadaghiani, J. W. Taylor, and K. Cummings, Positive attitudinal shifts with the Physics by Inquiry curriculum across multiple implementations, Phys. Rev. ST Phys. Educ. Res. 8, 010102 (2012).

[27] W. K. Adams, K. K. Perkins, N. S. Podolefsky, M. Dubson, N. D. Finkelstein, and C. E. Wieman, New instrument for measuring student beliefs about phsics and learning physics: The Colorado Learning Attitudes about Science Survey, Phys. Rev. ST Phys. Educ. Res. 2, 010101 (2006).

[28] D. Hammer, Epistemological beliefs in introductory physics, Cognit. Instr. 12, 151 (1994).

[29] L. Ding, Verification of casual influences of reasoning skills and epistemology on physics conceptual learning, Phys. Rev. ST Phys. Educ. Res. 10, 023101 (2014).

[30] K. A. Douglas, M. S. Yale, D. E. Bennett, M. P. Haugan, and L. A. Bryan, Evaluation of Colorado Learning Attitudes about Science Survey, Phys. Rev. ST Phys. Educ. Res. 10, 020128 (2014).

[31] M. A. McDaniel, M. J. Cahill, R. F. Frey, M. Rauch, J. Doele, D. Ruvolo, and M. M. Daschbach, Individual differences in learning exemplars versus abstracting rules: Associations with exam performance in college science, J. Appl. Res. Mem. Cogn. 7, 241 (2018).

[32] G. Pólya, How to Solve It: A New Aspect of Mathematical Method, 2nd ed. (Doubleday, New York, 1957).

[33] A. Newell and H. A. Simon, Human Problem Solving (Prentice-Hall, New Jersey, 1972).

[34] P. F. Delaney, K. A. Ericsson, and M. E. Knowles, Immediate and sustained effects of planning in a problem-solving task, J. Exp. Psychol. Learn. Mem. Cogn. 30, 1219 (2004).

[35] J.H. Larkin and F. Reif, Understanding and teaching problem-solving in physics, Eur. J. Sci. Educ. 1, 191 (1979).

[36] B. H. Ross, J. P. Mestre, and J. L. Docktor, Understanding how to teach physics understanding, in Integrating Cognitive Science with Innovative Teaching in STEM Disciplines, edited by M. A. McDaniel, R. Frey, S. Fitzpatrick, and H. L. Roediger (Washington University Libraries, St. Louis, MO, 2014), https://doi.org/10.7936/K75Q4T1X.

[37] M. E. Atwood and P. G. Polson, A process model for water jug problems, Cogn. Psychol. 8, 191 (1976).

[38] M. A. McDaniel and M. S. Schlager, Discovery learning and transfer of problem-solving skills, Cognit. Instr. 7, 129 (1990).

[39] C. Tenison, J. M. Fincham, and J. R. Anderson, Phases of learning: How skill acquisition impacts cognitive processing, Cogn. Psychol. 87, 1 (2016).

[40] R. R. Hake, Interactive-engagement versus traditional methods: A six-thousand-student survey of mechanics test data for introductory physics courses, Am. J. Phys. 66, 64 (1998).

[41] T. R. Coyle, J. M. Purcell, A. C. Snyder, and P. Kochunov, Non- $g$ residuals of the SAT and ACT predict specific abilities, Intelligence 41, 114 (2013).

[42] T. Moore, Six Ideas That Shaped Physics, 2nd ed. (McGraw-Hill, New York, 2007).

[43] C. H. Crouch, J. Watkins, A. P. Fagen, and E. Mazur, Peer Instruction: Engaging Students one-on-one, all at once, in Research-Based Reform of University Physics, edited by E.F. Redish and P. J. Cooney, Reviews in PER Vol. 1 (2007), http://www.per-central.org/document/ ServeFile.cfm?ID=4990.

[44] R. W. Schvaneveldt, F. T. Durso, T. E. Goldsmith, T. B. Bree, N. M. Cooke, and J.C. De Maio, Measuring the structure of expertise, Int. J. Man. Mach. Stud. 23, 699 (1985).

[45] R. W. Schvaneveldt, Pathfinder Associative Networks (Ablex Publishing Corporation, Norwood, NJ, 1990).

[46] R. W. Schvaneveldt and F. T. Durso, General semantic networks, in Proceedings of the Annual Meeting of the Psychonomic Society, Philadelphia, PA (1981).

[47] J. Cohen, Statistical Power Analysis for the Behavioral Sciences, 2nd ed. (Erlbaum, Hillsdale, NJ, 1988).

[48] J. L. Docktor, J. Dornfeld, E. Frodermann, K. Heller, L. Hsu, K. A. Jackson, A. Mason, Q. X. Ryan, and J. Yang, Assessing student written problem solutions: A problemsolving rubric with application to introductory physics, Phys. Rev. Phys. Educ. Res. 12, 010130 (2016).

[49] R. J. Dufresne, W. J. Gerace, P. T. Hardiman, and J. P. Mestre, Constraining novices to perform expertlike problem analyses: Effects on schema acquisition, J. Learn. Sci. 2, 307 (1992).

[50] P. T. Hardiman, R. Dufresne, and J. P. Mestre, The relation between problem categorization and problem solving among novices and experts, Mem. Cogn. 17, 627 (1989).

[51] J. Mestre, R. J. Dufresne, W. J. Gerace, P. T. Hardiman, and J. S. Touger, Promoting skilled problem solving behavior among beginning physics students, J. Res. Sci. Teach. 30, 303 (1993).

[52] M. T. H Chi, P. Feltovich, and R. Glaser, Categorization and representation of physics problems by experts and novices, Cogn. Sci. 5, 121 (1981).

[53] J. L. Docktor, J. P. Mestre, and B. H. Ross, Impact of a short intervention on novices' categorization criteria, Phys. Rev. ST Phys. Educ. Res. 8, 020102 (2012). 